\title{
The Royal College of Ophthalmologists' National Ophthalmology Database study of cataract surgery: Report 7, immediate sequential bilateral cataract surgery in the UK: Current practice and patient selection
}

\author{
John C. Buchan (10 ${ }^{1,2} \cdot$ Paul H. J. Donachie ${ }^{3,4} \cdot$ Andy Cassels-Brown ${ }^{5,6} \cdot$ Christopher Liu $^{7} \cdot$ Andrew Pyott $^{6}$. \\ Jennifer L. Y. Yip $\mathbb{D}^{1} \cdot$ Mehran Zarei-Ghanavati $^{8} \cdot$ John M. Sparrow $^{3,9}$
}

Received: 11 July 2019 / Revised: 18 December 2019 / Accepted: 19 December 2019 / Published online: 7 January 2020

(c) The Author(s), under exclusive licence to The Royal College of Ophthalmologists 2020

\begin{abstract}
Background Cataract extraction is the most frequently performed surgical intervention in the world and demand is rising due to an ageing demography. One option to address this challenge is to offer selected patients immediate sequential bilateral cataract surgery (ISBCS). This study aims to investigate patient and operative characteristics for ISBCS and delayed bilateral cataract surgery (DSCS) in the UK.

Methods Data were analysed from the Royal College of Ophthalmologists' National Ophthalmology Database Audit (NOD) of cataract surgery. Eligible patients were those undergoing bilateral cataract extraction from centres with a record of at least one ISBCS operation between 01/04/2010 and 31/08/2018. Variable frequency comparison was undertaken with chi-square tests.

Results During the study period, 1073 patients had ISBCS and 248,341 DSCS from 73 centres. A higher proportion of ISBCS patients were unable to lie flat $(11.3 \%$ vs. $1.8 \% ; p<0.001)$, unable to cooperate $(9.7 \%$ vs. $2.7 \% ; p<0.001)$; underwent general anaesthesia $(58.7 \%$ vs. $6.6 \%(p<0.001))$; had brunescent/white/mature cataracts (odds ratio (OR) 5.118 ); no fundal view/vitreous opacities (OR 8.381); had worse pre-operative acuity 0.60 LogMAR ISBCS vs. 0.50 (first) and 0.40 (second eye) DSCS and were younger (mean ages, 71.5 vs. 75.6 years; $p<0.001$ ). Posterior capsular rupture $(\mathrm{PCR})$ rates adjusted for case complexity were comparable (0.98\% ISBCS and $0.78 \%$ DSCS).

Conclusions ISBCS was performed on younger patients, with difficulty cooperating and lying flat, worse pre-operative vision, higher rates of known PCR risk factors and more frequent use of general anaesthesia than DSCS in centres recorded on NOD.
\end{abstract}

Supplementary information The online version of this article (https:// doi.org/10.1038/s41433-019-0761-z) contains supplementary material, which is available to authorized users.

John C. Buchan

John.buchan@1shtm.ac.uk

1 International Centre for Eye Health, London School of Hygiene and Tropical Medicine, London, UK

2 Leeds Teaching Hospitals NHS Trust, Beckett Street, Leeds LS9 7TF, UK

3 The Royal College of Ophthalmologists' National Ophthalmology Audit, 18 Stephenson Way, London NW1 2HD, UK

4 Gloucestershire Hospitals NHS Foundation Trust,

\section{Introduction}

Cataract surgery is the most common surgical intervention in the world, and almost half a million cataract operations are now done each year in the UK $(\approx 430,000$ NHS cataracts

Cheltenham GL53 7AN, UK

5 Centre for Sustainable Healthcare, Oxford OX2 7JQ, UK

6 NHS Highland, Inverness IV2 7GE, UK

7 Tongdean Eye Clinic. Sussex Eye Hospital, Brighton and Sussex University Hospitals NHS Trust, Brighton and Sussex Medical School, Eastern Road, Brighton BN2 5BF, UK

8 Eye Research Center, Farabi Eye Hospital, Tehran University of Medical Sciences, Tehran, Iran

9 Bristol Eye Hospital, Lower Maudlin Street, Bristol BS1 2LX, UK 
in England and Wales 2017/18). Cataract surgery is established as a highly cost-effective intervention with substantial quality of life gains on offer for patients, both for first and second eye surgeries [1]. It has been suggested that offering patients surgery for both eyes on the same day, termed immediate sequential bilateral cataract surgery (ISBCS), could achieve full quality of life gains faster, whilst more effectively using the finite financial, environmental and human resources available to the UK NHS.

Taking an estimate of $£ 1000$ per completed one-eye cataract pathway, the NHS spend is therefore nearing $£ 500$ million annually [2]. With an aging population, this cost is set to increase by $50 \%$ in the next 20 years [3]; however, a $50 \%$ increase in either the financial or the human resources available to deliver that service is not widely anticipated. Unless there are substantial changes to current NHS working practices, rationing of cataract surgery will be inevitable, either explicitly by setting treatment thresholds or covertly, by waiting lists.

Rationing of cataract surgery in the UK is already occurring [4,5]. This is true despite the 2017 NICE guidance on adult cataract management [NG77] stating that rationing and delay of surgery once sight impairment from cataract has been identified is not justifiable on cost or clinical grounds [6].

If we are to mitigate the effects of the demographic time bomb [7], encouraging providers to offer ISBCS potentially offers a more cost-effective and sustainable pathway, with reduced outpatient visits, enhanced operating theatre efficiency through shorter surgical turnaround times between operations, and reduced carbon footprint [8].

The NICE Cataract Guidelines [NG77] suggest that clinicians may consider offering ISBCS to two groups of patients with bilateral cataract:

people who are at low risk of ocular complications during and after surgery.

people who need to have general anaesthesia for cataract surgery but for whom general anaesthesia carries an increased risk of complications or distress [6].

Previous published UK case series exist of patients in the high-risk GA category being offered ISBCS [9], but there is no published evidence regarding how widespread this practice is presently, and whether ISBCS is being offered to patients in the low-risk category-who would be expected to form the majority of the half million eyes operated for cataract each year.

This analysis of data submitted to the Royal College of Ophthalmologists' National Ophthalmology Database Audit (RCOphth NOD) [10], aims to describe current practice in ISBCS provision in contributing centres as representative of English and Welsh ophthalmic departments. Differences between patients with bilateral cataract undergoing ISBCS and those undergoing delayed sequential surgery (DSCS) are described, so as to typify current ISBCS patients and evaluate how current practice reflects the NICE guidance.

\section{Methods}

The RCOphth NOD receives anonymised data from participating NHS Trusts in England, Health Boards in Wales and Independent Sector Treatment Centres (ISTC) providing NHS Funded cataract surgery in England, Wales and one ISTC in Guernsey. The data are recorded on electronic medical record systems (EMR) or in-house databases and extracted in the autumn of each year for cataract operations using phacoemulsification to treat patients aged 18 years or older, where the primary intention was cataract surgery and not combined 'cataract + other' surgery, unless the 'other' surgery formed part of the cataract operation (e.g. an operative manoeuvre to increase the size of the pupil). Further information on an audit eligible cataract operation can found on the audit website (www.nodaudit.org.uk).

Centres contributing to the RCOphth NOD may reasonably be taken as a surrogate for practice in England and Wales as a whole, with the last data extraction providing 216,000 operations from 101 centres, well over half $(\sim 60 \%)$ of the traditional NHS providers.

The analysis presented in this paper concerns cataract operations satisfying all bar the centre location eligibility criteria that applies to National Ophthalmology Audit (NOA), from any RCOphth NOD contributing centre with at least $50 \mathrm{NOA}$ eligible cataract operations performed between $01 / 04 / 2010$ and 31/08/2018. Only centres with a record of at least one ISBCS operation where both eye operations satisfied the NOA eligibility criteria during the study period are included. For comparison, DSCS patients are those from these centres who had both eyes undergo NOD eligible cataract surgery where the second eye surgery was conducted within 1 year of the first. The data were recorded on either the Medisoft EMR system (Medisoft Ophthalmology, Medisoft Limited, Leeds, UK, www.medisoft.co.uk), the Open Eyes EMR system (www.openeyes.org.uk), or 'in-house' data collection systems compliant with the National Cataract Dataset [11].

Pre-operative visual acuity (VA) was defined as the best recorded distance VA (corrected or uncorrected but not pinhole) that is closest to the date of surgery, including the day of surgery and within 6 months prior to surgery. For numeric calculations, the extreme low vision estimates from the LogMAR chart representing count fingers $(\mathrm{CF})$, hand movements (HM), perception of light (PL) and no perception of light (NPL) are replaced with 2.10, 2.40, 2.70, and 3.00 , respectively. 
Posterior capsular rupture (PCR) was defined as in the NOA and case complexity expected and adjusted rates were calculated using the case complexity adjustment from the NOA, where full details about the adjustment can be found on the audit website (www.nodaudit.org.uk). The variables used for case complexity adjustment are the surgeon grade, first/second eye surgery and all variables marked with (a) in Tables 1 and 2. For the ISBCS patients, both eyes are assumed to be the first treated eye in the case complexity adjustment.

Comparisons at the patient level are performed between ISBCS patients and at the first eye surgery for DSCS patients using $\chi^{2}$ tests except for the comparison of the patient's age which was performed using the student's $t$ test with the Welch adjustment. Comparisons at the eye level were performed using univariate logistic regression with cluster adjustment for the standard errors where the patients are considered as the clusters. All statistical tests were two sided.

The lead clinician and Caldicott Guardian (responsible nominee for data protection) at each centre provided written approval for anonymised data extraction. Anonymized database analyses of this type do not require ethical permission due to being viewed as audit or service evaluation (see http://www.hra.nhs.uk/research-community/beforeyou-apply/determinewhether-your-study-is-research/). This study was conducted in accordance with the declaration of Helsinki, and the UK's Data Protection Act.

\section{Results}

\section{Patient demographics}

During the study period, 1073 patients had ISBCS and 248,341 patients DSCS from 73 centres; thus from centres doing any ISBCS in the study period, $0.43 \%$ were ISBCS.

The median time between the first eye and second eye surgery for the DSCS patients was 3.4 months. The second eye surgery was performed within 3 months of the first eye surgery for 110,337 (44.4\%) DSCS patients and within 6 months for 209,839 (84.5\%) DSCS patients.

Of the ISBCS patients, $671(62.5 \%)$ were female vs. $149,076(60.0 \%)$ of DSCS patients $(p=0.160)$. Using the first eye surgery of the DSCS patients as the comparator, the ISCBS patients tended to be younger than the DSCS
Table 1 Demographics for the ISBCS and DSCS patients.

\begin{tabular}{|c|c|c|c|c|}
\hline$N($ Column \%) & ISBCS & DSCS & Overall & $p$ value \\
\hline Number of patients & 1073 & 248,341 & 249,414 & - \\
\hline \multicolumn{5}{|l|}{ Patients gender ${ }^{\mathrm{a}}$} \\
\hline Male & $402(37.5)$ & 99,037 (39.9) & 99,439 (39.9) & \multirow[t]{3}{*}{0.160} \\
\hline Female & $671(62.5)$ & $149,076(60.0)$ & $149,747(60.0)$ & \\
\hline Not recorded & $0(0.0)$ & $228(<0.1)$ & $228(<0.1)$ & \\
\hline \multicolumn{5}{|l|}{ Patients age (years) ${ }^{\mathrm{a}, \mathrm{b}}$} \\
\hline Median & 74.6 & 76.9 & 76.9 & \multirow[t]{3}{*}{-} \\
\hline Inter-quartile range & $62.7-82.2$ & $70.0-82.4$ & $70.0-82.4$ & \\
\hline Range & $21.4-100.6$ & $18.0-112.5$ & $18.0-112.5$ & \\
\hline Mean & 71.5 & 75.6 & 75.5 & $<0.001$ \\
\hline Standard deviation & 14.4 & 9.7 & 9.8 & - \\
\hline$<70$ & $424(39.5)$ & $62,009(25.0)$ & $62,433(25.0)$ & \multirow[t]{6}{*}{$<0.001$} \\
\hline $70-74$ & $127(11.8)$ & $42,508(17.1)$ & $42,635(17.1)$ & \\
\hline $75-79$ & $181(16.9)$ & $54,901(22.1)$ & $55,082(22.1)$ & \\
\hline $80-84$ & $166(15.5)$ & $51,186(20.6)$ & $51,352(20.6)$ & \\
\hline $85-89$ & $114(10.6)$ & $28,959(11.7)$ & $29,073(11.7)$ & \\
\hline$\geq 90$ & $61(5.7)$ & $8778(3.5)$ & $8839(3.5)$ & \\
\hline \multicolumn{5}{|l|}{ Patient with the following ${ }^{\mathrm{b}}$} \\
\hline Diabetes mellitus & $153(14.3)$ & $45,070(18.1)$ & $45,223(18.1)$ & $<0.001$ \\
\hline Could not lie flat during surgery ${ }^{a}$ & $121(11.3)$ & $4496(1.8)$ & $4617(1.9)$ & $<0.001$ \\
\hline Could not cooperate with the surgery & 104 (9.7) & $6674(2.7)$ & 6778 (2.7) & $<0.001$ \\
\hline
\end{tabular}

Statistical comparisons performed using $\chi^{2}$ tests except for the comparison of the patient's age which was performed using the student's $t$ test with the Welch adjustment

${ }^{a}$ These conditions are included in the case complexity adjustment for PCR

${ }^{b}$ These estimates for the DSCS patients are at the time of their first eye cataract surgery, and some of these could be different at the time of their second eye cataract surgery 
Table 2 Ocular co-pathology and known risk factors for the ISBCS and DSCS operations.

\begin{tabular}{|c|c|c|c|c|c|c|c|}
\hline \multirow[t]{2}{*}{$N($ Column \%) } & \multirow{2}{*}{$\begin{array}{l}\text { ISBCS eyes } \\
(N=2146)\end{array}$} & \multirow{2}{*}{$\begin{array}{l}\text { DSCS eyes } \\
(N=496,682)\end{array}$} & \multirow[t]{2}{*}{ OR } & \multirow[t]{2}{*}{$95 \% \mathrm{CI}$ for OR } & \multirow[t]{2}{*}{$p$ value } & \multicolumn{2}{|c|}{$\%$ of DSCS eyes at their: } \\
\hline & & & & & & $\begin{array}{l}\text { First eye } \\
\text { surgery }\end{array}$ & $\begin{array}{l}\text { Second eye } \\
\text { surgery }\end{array}$ \\
\hline $\begin{array}{l}\text { No ocular co-pathology or know } \\
\text { risk factors }\end{array}$ & $1134(52.8)$ & $306,356(61.7)$ & 1.436 & $1.282-1.610$ & $<0.001$ & 59.4 & 64.0 \\
\hline $\begin{array}{l}\text { At least one ocular co-pathology or } \\
\text { known risk factor }\end{array}$ & $1012(47.2)$ & $190,326(38.3)$ & & & & 40.6 & 36.0 \\
\hline \multicolumn{8}{|l|}{ The presence of } \\
\hline Age-related macular degeneration & $147(6.9)$ & $47,123(9.5)$ & 0.702 & $0.558-0.882$ & 0.002 & 9.6 & 9.3 \\
\hline Amblyopia $^{\mathrm{a}}$ & $34(1.6)$ & 7835 (1.6) & 1.004 & $0.690-1.462$ & 0.982 & 1.7 & 1.4 \\
\hline $\begin{array}{l}\text { Brunescent/white/mature } \\
\text { cataract }^{\mathrm{a}}\end{array}$ & $319(14.9)$ & $16,385(3.3)$ & 5.118 & $4.367-5.999$ & $<0.001$ & 4.2 & 2.4 \\
\hline Corneal pathology & $62(2.9)$ & $14,142(2.8)$ & 1.015 & $0.725-1.421$ & 0.930 & 3.0 & 2.7 \\
\hline Diabetic retinopathy $^{\mathrm{a}}$ & $113(5.3)$ & $25,299(5.1)$ & 1.036 & $0.799-1.342$ & 0.791 & 5.1 & 5.1 \\
\hline Glaucoma & $152(7.1)$ & $39,346(7.9)$ & 0.886 & $0.706-1.113$ & 0.298 & 8.1 & 7.7 \\
\hline High myopia $^{a}$ & $139(6.5)$ & $24,034(4.8)$ & 1.362 & $1.074-1.727$ & 0.011 & 5.3 & 4.4 \\
\hline Inherited eye diseases & $31(1.4)$ & $662(0.1)$ & 10.982 & $6.616-18.229$ & $<0.001$ & 0.1 & 0.1 \\
\hline $\begin{array}{l}\text { No fundal view/vitreous } \\
\text { opacities }^{\mathrm{a}}\end{array}$ & $142(6.6)$ & $4164(0.8)$ & 8.381 & $6.647-10.568$ & $<0.001$ & 1.3 & 0.4 \\
\hline Other macular pathology & $25(1.2)$ & $8522(1.7)$ & 0.675 & $0.424-1.076$ & 0.099 & 1.8 & 1.7 \\
\hline Other retinal vascular pathology & $13(0.6)$ & $3416(0.7)$ & 0.880 & $0.440-1.761$ & 0.718 & 0.7 & 0.7 \\
\hline Optic nerve/CNS disease & $24(1.1)$ & $1705(0.3)$ & 3.283 & $1.900-5.675$ & $<0.001$ & 0.4 & 0.3 \\
\hline Pseudoexfoliation/Phacodenesis ${ }^{\mathrm{a}}$ & $21(1.0))$ & $4100(0.8)$ & 1.187 & $0.671-2.102$ & 0.556 & 0.9 & 0.8 \\
\hline Previous retinal detachment & $9(0.4)$ & $2079(0.4)$ & 1.002 & $0.486-2.067$ & 0.996 & 0.6 & 0.2 \\
\hline Previous vitrectomy & $20(0.9)$ & $3538(0.7)$ & 1.311 & $0.764-2.249$ & 0.325 & 1.0 & 0.4 \\
\hline Previous trabeculectomy ${ }^{\mathrm{a}}$ & $3(0.1)$ & $1439(0.3)$ & 0.482 & $0.155-1.497$ & 0.207 & 0.3 & 0.3 \\
\hline Uveitis/Synaechiae & $13(0.6)$ & $2923(0.6)$ & 1.029 & $0.483-2.196$ & 0.940 & 0.7 & 0.5 \\
\hline $\begin{array}{l}\text { Unspecified 'other' ocular co- } \\
\text { pathology }\end{array}$ & $148(6.9)$ & $26,896(5.4)$ & 1.294 & $1.034-1.619$ & 0.024 & 6.0 & 4.9 \\
\hline
\end{tabular}

$\mathrm{CI}=$ confidence interval

${ }^{a}$ These conditions are included in the case complexity adjustment for PCR

patients, mean ages 71.5 years vs. 75.6 years $(p<0.001)$ (Fig. 1), and $39.5 \%$ of ISBCS patients were aged $<70$ years old vs. $25.0 \%$ of DSCS patients. A lower proportion of ISBCS patients had diabetes mellitus than DSCS patients $(14.3 \%$ vs. $18.1 \%, p<0.001)$. More ISBCS patients were unable to lie flat during surgery than DSCS patients $(11.3 \%$ vs. $1.8 \%, p<0.001)$, and more ISBCS patients were unable to cooperate during surgery than DSCS patients $(9.7 \%$ vs. $2.7 \%, p<0.001)$, Table 1 . The distribution of patients for ISBCS and DSCS across the national deciles of area deprivation are presented in the Supplementary information online (Supplementary table 1).

\section{Operative details}

General anaesthesia was administered to 630 (58.7\%) ISBCS patients vs. 16,429 (6.6\%) DSCS patients (either operation) $(p<0.001)$. The 2146 ISBCS operations were performed by 431 individual surgeons, where consultant surgeons performed $1815(84.6 \%)$ of the operations and trainee surgeons 331 (15.4\%) operations and for 997 (92.9\%) patients (1994 operations) both ISBCS operations were performed by the same surgeon.

\section{Ocular co-pathology and known PCR risk factors}

At least one ocular co-pathology or known risk factor for PCR was present in 1012 (47.2\%) ISBCS eyes and 190,326 (38.3\%) DSCS eyes, where the OR for the higher rate in ISBCS eyes was 1.436 (95\% CI: 1.282-1.610; $p<0.001)$.

Brunescent/white/mature cataracts were recorded for $14.9 \%$ ISBCS eyes vs. 3.3\% DSCS eyes, OR 5.118 (95\% CI: $4.367-5.999 ; p<0.001)$. No fundal view/vitreous opacities were recorded for $6.6 \%$ ISBCS eyes vs. $0.8 \%$ DSCS eyes, OR 8.381 (95\% CI: 6.647-10.568; $p<0.001)$. The other ocular co-pathology or known PCR risk factors that 


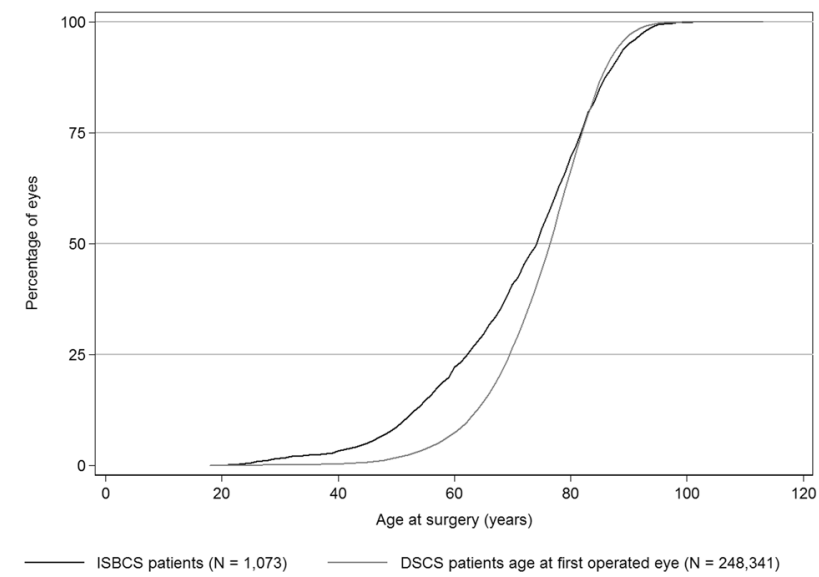

Fig. 1 Cumulative frequency of the patient's age in years: $N=1073$ patients treated with ISBCS and 248,341 patients treated with DSCS at the time of their first cataract surgery.

were statistically more frequently recorded for ISBCS eyes than DSCS eyes were high myopia, optic nerve/CNS disease, unspecified 'other' and inherited eye diseases which although had a massive OR of 10.982 is one of the rarest conditions and would include different very rare inherited diseases. Age-related macular degeneration was the only ocular co-pathology or known PCR risk factor that was more frequently recorded for DSCS eyes than ISBCS eyes, consistent with the younger age of the ISBCS patients.

No individual ocular co-pathology or known PCR risk factor was more frequently recorded for the second eye operation than the first eye operation for the DSCS patients. The following ocular co-pathologies/risk indicators for PCR were more frequently recorded by $>0.5 \%$ points in DSCS patients first eye operation than their second eye operation; brunescent/white/mature cataract, high myopia, no fundal view/vitreous opacities, previous vitrectomy and unspecified 'other', Table 2.

\section{Operative complications}

An intra-operative complication occurred in 76 (3.5\%) ISBCS eyes and 12,792 (2.6\%) DSCS eyes, with an OR of 1.389 (95\% CI: $1.082-1.782 ; p=0.010$ ) for the higher rate in ISBCS eyes than DSCS eyes. For the DSCS patients, an intra-operative complication occurred in $6230(2.5 \%)$ first eye operations and $6562(2.6 \%)$ second eye operations.

PCR occurred in $41(1.9 \%)$ ISBCS eyes and $5720(1.2 \%)$ DSCS eyes with an OR of 1.672 (95\% CI: 1.220-2.290; $p=0.001$ ) for the higher rate in ISBCS eyes than DSCS eyes. For the DSCS patients, PCR occurred in $2612(1.1 \%)$ first eye operations and 3108 (1.3\%) second eye operations. The expected rate PCR (adjusted according to the frequency of known risk factors in those patients) was $2.15 \%$ (95\% CI: $1.61-2.85 \%)$ for ISBCS eyes and $1.63 \%$ (95\% CI:
$1.60-1.67 \%)$ for DSCS eyes, and the case complexity adjusted rate of PCR was $0.98 \%$ (95\% CI: $0.64-1.49 \%$ ) for ISBCS eyes and $0.78 \%$ (95\% CI: $0.75-0.80 \%$ ) for DSCS eyes, both lower than the overall consultant rate of $1.1 \%$ used in The National Cataract Audit.

\section{Post-operative complications}

For reasons discussed below, detailed reporting and analysis of post-operative complications have not been undertaken, but there were no reports of post-surgery endophthalmitis in the ISBCS group, and $53(0.01 \%)$ for the DSCS group.

\section{Pre-operative visual acuity}

A pre-operative VA measurement was recorded for 1790 (83.4\%) ISBCS eyes, where the median pre-operative VA was 0.60 LogMAR (6/24 Snellen equivalent). For 418 (23.4\%) ISBCS eyes the pre-operative VA was CF or worse and the pre-operative VA distribution was very similar for ISBCS patients left and right eyes.

For the DSCS patients first eye operation, a pre-operative VA was recorded for $236,304(95.2 \%)$ eyes, where the median pre-operative VA was 0.50 LogMAR (6/19 Snellen equivalent) and for 11,893 (5.0\%) eyes the pre-operative VA was CF or worse.

For the DSCS patients second eye operation, a preoperative VA was recorded for 229,564 (92.4\%) eyes, and in contrast to the first eye operation the pre-operative VA profile was generally better for the second eye operation, where the median pre-operative VA was 0.40 LogMAR (6/15 Snellen equivalent) and for 7025 (3.1\%) eyes the preoperative VA was CF or worse, Fig. 2 and Table 3. The recording of a pre-operative vision of NPL for some patients (Table 3 ) is presumed to represent either cosmetic or therapeutic extractions, or a reflection of the fact that relatively less-trained members of the multi-disciplinary team often record visual acuities on the EMR, and that the ophthalmologist presumably omits to correct this recording when they subsequently examine the patient and find PL to be present prior to offering surgery.

\section{Discussion}

For over three decades, articles in peer reviewed journals have asked if ISBCS is a practice the NHS should be adopting as routine [12-14]. Despite this interest, our study demonstrates that ISBCS is being predominantly performed just for a small number of higher complexity patients, evidenced by their issues with cooperating, lying flat, higher rates of brunescent/white/mature cataracts and no fundal view/vitreous opacities. 
Relating to current NICE guidance, the preponderance of these factors in the ISBCS groups and the finding that nearly $60 \%$ of ISBCS operations were carried out under GA suggests that ISBCS is being offered to "people who need to have general anaesthesia for cataract surgery but for whom general anaesthesia carries an increased risk of complications or distress." The other category for whom NICE suggest consideration of ISBCS be given, "people who are at low risk of ocular complications during and after surgery", who would be expected to constitute the majority of those undergoing surgery for bilateral lens opacity, are therefore shown to be either not offered this surgical option, or to be universally declining it.

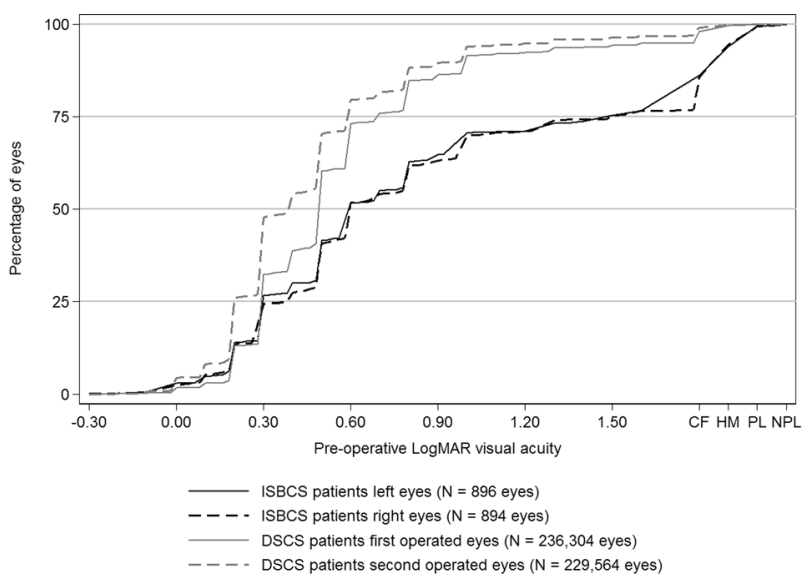

Fig. 2 Cumulative frequency of LogMAR pre-operative visual acuity for ISBCS patients left and right eyes and DSCS patients first and second treated eyes: $N=896$ ISBCS patients left eyes, 894 ISBCS patients right eyes, 236,304 DSCS patients first eye surgery and 229,564 DSCS patients second eye surgery.
It is possible that ISBCS is being offered to low-risk patients by surgeons who are not yet contributing their data to the RCOphth NOD. It is also possible that data entry into the EMR that contribute to the NOD is inaccurate, as neither triangulation nor quality control of that data is undertaken. However, based on two recent surveys undertaken to identify uptake of ISBCS as a surgical option in the UK ophthalmology departments it seems unlikely that the NOD data are unrepresentative of UK practice. In 2016, UK lead clinicians were surveyed by email using the RCOphth database; no department reported offering ISBCS to routine patients from 42 responses, and only 14\% (6/42) reported that they would offer ISBCS to individual patients according to specific need (unpublished data-personal communication (A. AzuaraBlanco). The same finding was reported from the RCOphth Way Forward project which requested phone interviews with consultants nominated as leading cataract services in their units; 50 consultants from across the UK were interviewed (representing 50/142 UK NHS eye departments) and none were found to be offering ISBCS routinely [7].

Unless both of these surveys and NOD data recruitment suffer from the same systematic biases, the assertion that the RCOphth NOD data reflect the UK national practice of ISBCS seems reasonable. Internationally, there has been adoption of ISBCS in a small number of countries as a mainstream option for patients with bilateral cataract, particularly in areas of lower population density where travel times are substantial (parts of Scandinavia, Spain and Canada) [15]. Experience from those settings has been encouraging but the evidence base has not proved sufficiently strong to convince either NICE or UK ophthalmologists to feel confident to make ISBCS widely available to patients.
Table 3 Pre-operative LogMAR visual acuity.

\begin{tabular}{|c|c|c|c|c|c|}
\hline \multirow[b]{2}{*}{$N(\%)$} & \multicolumn{2}{|l|}{ ISBCS patients } & \multicolumn{2}{|l|}{ DSCS patients } & \multirow[t]{2}{*}{ Overall } \\
\hline & Left eyes & Right eyes & First eye surgery & $\begin{array}{l}\text { Second eye } \\
\text { surgery }\end{array}$ & \\
\hline Number of eyes & 1073 & 1073 & 248,341 & 248,341 & 498,828 \\
\hline $\begin{array}{l}\text { Number without } \\
\text { VA data }\end{array}$ & $177(16.5)$ & $179(16.7)$ & $12,037(4.8)$ & 18,777 (7.6) & $31,170(6.2)$ \\
\hline $\begin{array}{l}\text { Number with } \\
\text { VA data }\end{array}$ & $896(83.5)$ & $894(83.3)$ & $236,304(95.2)$ & $229,564(92.4)$ & $467,658(93.8)$ \\
\hline Median & 0.60 & 0.60 & 0.50 & 0.40 & 0.50 \\
\hline $\begin{array}{l}\text { Inter- } \\
\text { quartile range }\end{array}$ & $0.30-1.50$ & $0.40-1.50$ & $0.30-0.70$ & $0.20-0.60$ & $0.30-0.64$ \\
\hline Range & $-0.20-\mathrm{NPL}$ & $-0.30-\mathrm{NPL}$ & $-0.30-\mathrm{NPL}$ & $-0.30-\mathrm{NPL}$ & $-0.30-\mathrm{NPL}$ \\
\hline \multicolumn{6}{|l|}{ Number with } \\
\hline $\mathrm{CF}$ & 87 (9.7) & $80(8.9)$ & $7420(3.1)$ & $5114(2.2)$ & $12,701(2.7)$ \\
\hline HM & $70(7.8)$ & $79(8.8)$ & $3544(1.5)$ & $1626(0.7)$ & 5319 (1.1) \\
\hline PL & $49(5.5)$ & $44(4.9)$ & $871(0.4)$ & $267(0.1)$ & $1231(0.3)$ \\
\hline NPL & $4(0.4)$ & $5(0.6)$ & $58(<0.1)$ & $18(<0.1)$ & $85(<0.1)$ \\
\hline
\end{tabular}


The decision to offer or not offer ISBCS is made by individual ophthalmologists. Other than reducing theatre turnaround time, there is no incentive for the surgeon to make ISBCS available as it creates an administrative burden to organise separate batch numbers for all consumables used intraoperatively. The lack of incentive is compounded by the perception of increased risk to the patient. Although there were no cases of endophthalmitis in the ISBCS group in this study, reports exist of clustering of endophthalmitis cases [16], which may not be entirely mitigated by separation of consumable batch numbers and instrument sterilisation timings. There is a further resource burden when general anaesthesia is used for surgery.

From the patients' perspective, there is some evidence that a prolonged gap between first and second eye surgery, in our series a median of 3.4 months, can increase the risk of falls [17], and diminish the quality of some patients' final visual function with poorer stereopsis, greater exophoria and reduced near point convergence [18]. On the other hand, it has been shown theoretically that delaying second eye surgery until the refractive outcome from the first eye is known may improve overall refractive outcome [19]. The loss of ability to alter refractive aim of the second eye is mentioned in the 2017 NICE cataract guidelines as something that needs to be told to patients opting for ISBCS. Despite this, superiority of refractive outcomes of DSBCS over ISBCS in large case series has not been demonstrated [20]. We also felt it unhelpful to deal with the issue of refractive outcomes here, as the patient group being operated for ISBCS, as indicated by the high GA rate, is not representative of the population that might in theory be offered ISBCS should this practice go to scale within in the NHS. The same reasons that led the surgeon to offer ISBCS in this series, (e.g. difficulty cooperating with surgery due to dementia or learning difficulties) would also likely mean there could be difficulty complying with subjective refraction. Despite arguments about the superiority of either pathway, the general assumption amongst the ophthalmic community is that the final outcome of bilateral cataract surgery will be the same whether the gap between the two operations is a few minutes or a few weeks - but the journey to that final outcome will differ.

The main strength of our study is that the data were nonselective, pooled and anonymized, so they may be more generalisable than data obtained from controlled trials. Although the study was a retrospective analysis, the data were collected prospectively as part of routine clinical practice. The advantages of EMR systems in clinical practice diminish if the use is not consistent for all parts of the clinical pathway. In our study this limitation affected the availability of data from post-surgery clinics and prevented us from investigating refractive outcomes and postoperative complications such as endophthalmitis. Due to this limitation, and the differences in many of the preoperative factors between the ISBCS and DSCS groups, we felt reporting of detailed post-operative comparison to be inappropriate.

It may be that the unsubstantiated perception of the absence of benefit to patients, along with the perception of increased risk of bilateral complications are insurmountable barriers unless better evidence is generated to inform the debate. Given the rarity of the most serious post-operative complications of cataract surgery (including non-ocular complications such as falls and road traffic accidents), it would not be possible to produce high-level evidence of the relative risks of different pathways. The question of patient benefit centres around the more rapid achievement of quality of life gains and avoidance of inter-operative imbalance, could however be informed by a randomised controlled trial, powered to cost-effectiveness, where effectiveness is defined in terms of patient centred outcomes and the area under curve of the improvement of quality of life generated by the surgical pathway. Further studies should include qualitative investigation of the perceptions of risk and how these are understood by patients and clinicians, along with exploration of the ethical issues of allowing patients to choose pathways with which some clinicians feel uncomfortable.

Unless there is a very large and currently unanticipated change in the global demographic, the treatment options for cataract, or the resources available to deliver health services, it is unavoidable that current cataract services will have to evolve. Sustainability is an increasingly important agenda for healthcare as a whole, and the environmental necessity to reduce our collective carbon footprint is pressing. ISBCS is an option that potentially offers benefits to patients, whilst meeting these challenges, but this study demonstrates that it is an option that has been largely ignored in the UK thus far.

\section{Summary}

\section{What was known before:}

NICE concluded that ophthalmologists may consider offering ISBCS to two groups of patients with bilateral cataract; high-risk general anaesthetic patients and lowrisk routine patients

In some high-income countries, ISBCS has become an established national pathway offered to patients Other than historic case series, there have not been any reports of ISBCS being performed at scale in the UK. 


\section{What this study adds:}

Less than $0.5 \%$ of the cataract operations performed in the UK between 2010 and 2018 were ISBCS

The case mix suggests that the majority of patients are offered ISBCS because they fall into the high-risk general anaesthesia category

ISBCS patients were younger, had more difficulties with cooperating and lying flat, worse pre-operative vision, and higher rates of known PCR risk factors than those on the delayed sequential cataract surgical pathway

This study provides evidence that no centre contributing to the NOD routinely performed ISBCS to treat low-risk bilateral cataracts between 2010 and 2018 .

Acknowledgements It is with deep regret that we note the death of our friend and colleague Robert Johnston, who sadly died in September 2016. Without his inspirational vision, determination and career long commitment to quality improvement in ophthalmology this work would not have been possible. We would like to acknowledge the support and guidance we have received from the National Audit Steering Committee which includes professional members, ophthalmologists and optometrists, and patient and public representatives with individual lay members as well as patient support groups being represented. We acknowledge the support of the hospitals that participated in this National Ophthalmology Database Audit study and thank our medical and non-medical colleagues for the considerable time and effort devoted to data collection. The participating centres included in this study are listed in alphabetic order below, separated into the 68 NHS Trusts or Health Boards from England and Cymru respectively, and the 5 Independent sector treatment centres including one from the Channel Islands. NHS Trusts in England: Barking, Havering and Redbridge University Hospitals NHS Trust; Barts Health NHS Trust; Blackpool Teaching Hospitals NHS Foundation Trust; Bradford Teaching Hospitals NHS Foundation Trust; Brighton and Sussex University Hospitals NHS Trust; Calderdale and Huddersfield NHS Foundation Trust; Chesterfield Royal Hospital NHS Foundation Trust; County Durham and Darlington NHS Foundation Trust; East Kent Hospitals University NHS Foundation Trust; East Suffolk and North Essex NHS Foundation Trust; East Sussex Healthcare NHS Trust; Epsom and St Helier University Hospitals NHS Trust; Frimley Health NHS Foundation Trust; Gloucestershire Hospitals NHS Foundation Trust; Great Western Hospitals NHS Foundation Trust; Hampshire Hospitals NHS Foundation Trust; Harrogate and District NHS Foundation Trust; Imperial College Healthcare NHS Trust; Isle of Wight NHS Trust; James Paget University Hospitals NHS Foundation Trust; King's College Hospital NHS Foundation Trust; Kingston Hospital NHS Foundation Trust; Leeds Teaching Hospitals NHS Trust; London North West University Healthcare NHS Trust; Manchester University NHS Foundation Trust; Mid Cheshire Hospitals NHS Foundation Trust; Moorfields Eye Hospital NHS Foundation Trust*; Norfolk and Norwich University Hospitals NHS Foundation Trust; North Cumbria University Hospitals NHS Trust; North West Anglia NHS Foundation Trust; Northern Devon Healthcare NHS Trust; Nottingham University Hospitals NHS Trust; Oxford University Hospitals NHS Foundation Trust; Portsmouth Hospitals NHS Trust; Royal Berkshire NHS Foundation Trust; Royal Cornwall Hospitals NHS Trust; Royal Free London NHS Foundation Trust; Royal Surrey County Hospital NHS Foundation Trust; Royal United Hospitals Bath NHS Foundation Trust; Salisbury NHS Foundation Trust; Sandwell and West Birmingham Hospitals NHS Trust; Sheffield Teaching
Hospitals NHS Foundation Trust; Sherwood Forest Hospitals NHS Foundation Trust; Shrewsbury and Telford Hospital NHS Trust; South Tees Hospitals NHS Foundation Trust; South Warwickshire NHS Foundation Trust; Southport and Ormskirk Hospital NHS Trust; St Helens and Knowsley Teaching Hospitals NHS Trust; Stockport NHS Foundation Trust; Surrey and Sussex Healthcare NHS Trust; The Hillingdon Hospitals NHS Foundation Trust; The Newcastle upon Tyne Hospitals NHS Foundation Trust; The Rotherham NHS Foundation Trust; The Royal Bournemouth and Christchurch Hospitals NHS Foundation Trust; United Lincolnshire Hospitals NHS Trust; University Hospital Southampton NHS Foundation Trust; University Hospitals Birmingham NHS Foundation Trust; University Hospitals Bristol NHS Foundation Trust; University Hospitals Coventry and Warwickshire NHS Trust; University Hospitals Plymouth NHS Trust; Warrington and Halton Hospitals NHS Foundation Trust; Wirral University Teaching Hospital NHS Foundation Trust; Wrightington, Wigan and Leigh NHS Foundation Trust; Wye Valley NHS Trust; Yeovil District Hospital NHS Foundation Trust; York Teaching Hospital NHS Foundation Trust University health boards in Cymru: Cardiff and Vale University LHB Independent sector treatment centres: Care UK (Emersons Green NHS Treatment Centre); Care UK (North East London NHS Treatment Centre); Care UK (Southampton NHS Treatment Centre); Medical specialists group Guernsey; SpaMedica (Manchester); SpaMedica (Newton-le-Willows) *Including Bedford Hospital NHS Trust and Croydon Health Services NHS Trust as these are part of the same governing authority for ophthalmology.

Funding This study was supported through The Healthcare Quality Improvement Partnership (HQIP) commissioned Royal College of Ophthalmologists (RCOphth) National Ophthalmology Database Audit, which is part of the National Clinical Audit and Patient Outcomes Programme (NCAPOP) and the Clinical Outcomes Programme (COP). The salary of JC Buchan is supported by the Queen Elizabeth Diamond Jubilee Trust through the Commonwealth Eye Health Consortium.

\section{Compliance with ethical standards}

Conflict of interest The authors declare that they have no conflict of interest.

Publisher's note Springer Nature remains neutral with regard to jurisdictional claims in published maps and institutional affiliations.

\section{References}

1. Shekhawat NS, Stock MV, Baze EF, Daly MK, Vollman DE, Lawrence MG, et al. Impact of first eye versus second eye cataract surgery on visual function and quality of life. Ophthalmology. 2017;124:1496-503.

2. National Ophthalmology Database 2017/18 https://www.nodaudit. org.uk/u/docs/20/twkmamtbwf/NOD\%20Full\%20Comprehensive $\% 20$ Report\%202019.pdf. 2019.

3. Buchan JC, Norman P, Shickle D, Cassels-Brown A, MacEwen C. Failing to plan and planning to fail. Can we predict the future growth of demand on UK Eye Care Services? Eye. 2019;33: 1029-31.

4. Coronini-Cronberg S, Lee H, Darzi A, Smith P. Evaluation of clinical threshold policies for cataract surgery among English commissioners. J Health Serv Res Policy. 2012;17:241-7.

5. Burdon M. End the postcode lottery for cataract surgery. BMJ. 2019;365:12293 
6. Cataracts in adults: management 2017. NICE guideline [NG77] https://www.niceorguk/guidance/ng77.

7. Buchan JC, Amoaku W, Barnes B, Cassels-Brown A, Chang BY, Harcourt J, et al. How to defuse a demographic time bomb: the way forward? Eye. 2017;31:1519-22.

8. Morris DS, Wright T, Somner JE, Connor A. The carbon footprint of cataract surgery. Eye. 2013;27:495-501.

9. Beatty S, Aggarwal RK, David DB, Guarro M, Jones H, Pearce JL. Simultaneous bilateral cataract extraction in the UK. Br J Ophthalmol. 1995;79:1111-4.

10. Day AC, Donachie PH, Sparrow JM, Johnston RL, Royal College of Ophthalmologists' National Ophthalmology D. The Royal College of Ophthalmologists' National Ophthalmology Database Study of cataract surgery: report 2, relationships of axial length with ocular copathology, preoperative visual acuity, and posterior capsule rupture. Eye. 2015;29:1528-37.

11. The Royal College of Ophthalmologists Informatics and Audit Sub-Committee. Dataset Guidelines. Version: 2.0. Oct 2018. The Royal College of Ophthalmologists. https://www.rcophth.ac.uk/w p-content/uploads/2018/11/Dataset-Guidelines.pdf.

12. Chang DF. Simultaneous bilateral cataract surgery. Br J Ophthalmol. 2003;87:253-4.

13. Smith GT, Liu CS. Is it time for a new attitude to "simultaneous" bilateral cataract surgery? Br J Ophthalmol. 2001;85:1489-96.
14. Fenton PJ, Gardner ID. Simultaneous bilateral intraocular surgery. Trans Ophthalmol Soc U K. 1982;102:298-301.

15. Arshinoff SA. Same-day cataract surgery should be the standard of care for patients with bilateral visually significant cataract. Surv Ophthalmol. 2012;57:574-9.

16. Pathengay A, Flynn HW Jr., Isom RF, Miller D. Endophthalmitis outbreaks following cataract surgery: causative organisms, etiologies, and visual acuity outcomes. J Cataract Refract Surg. 2012; 38:1278-82.

17. Meuleners LB, Fraser ML, Ng J, Morlet N. The impact of first- and second-eye cataract surgery on injurious falls that require hospitalisation: a whole-population study. Age Ageing. 2014;43:341-6.

18. Loba P, Rajska K, Simiera J, Wilczynski M, Omulecki W, Broniarczyk-Loba A. The influence of a prolonged interoperative period on binocular vision after bilateral cataract extractions. Eur J Ophthalmol. 2015;25:315-9.

19. Aristodemou P, Knox Cartwright NE, Sparrow JM, Johnston RL. First eye prediction error improves second eye refractive outcome results in 2129 patients after bilateral sequential cataract surgery. Ophthalmology. 2011;118:1701-9.

20. Herrinton LJ, Liu L, Alexeeff S, Carolan J, Shorstein NH. Immediate sequential vs. delayed sequential bilateral cataract surgery: retrospective comparison of postoperative visual outcomes. Ophthalmology. 2017;124:1126-35. 